\title{
Experimental study of isovector spin sum rules
}

\author{
A. Deur ${ }^{1}$, P. Bosted ${ }^{1}$, V. Burkert ${ }^{1}$, D. Crabb $^{2}$, V. Dharmawardane ${ }^{3 *}$, G. E. Dodge ${ }^{3}$, \\ T. A. Forest ${ }^{4}$, K. A. Griffioen ${ }^{5}$, S. E. Kuhn ${ }^{3}$, R. Minehart ${ }^{2}$, Y. Prok $^{2 \dagger}$ \\ ${ }^{1}$ Thomas Jefferson National Accelerator Facility, Newport News, VA 23606 \\ ${ }^{2}$ University of Virginia, Charlottesville, VA 22904 \\ ${ }^{3}$ Old Dominion University, Norfolk, VA 23529 \\ ${ }^{4}$ Idaho State University, Pocatello, ID, 83209 \\ ${ }^{5}$ College of William and Mary, Williamsburg, VA 23187
}

(Dated: March 5, 2022)

\begin{abstract}
We present the Bjorken integral extracted from Jefferson Lab experiment EG1b for $0.05<Q^{2}<$ $2.92 \mathrm{GeV}^{2}$. The integral is fit to extract the twist- 4 element $f_{2}^{p-n}$ which appears to be relatively large and negative. Systematic studies of this higher twist analysis establish its legitimacy at $Q^{2}$ around $1 \mathrm{GeV}^{2}$. We also performed an isospin decomposition of the generalized forward spin polarizability $\gamma_{0}$. Although its isovector part provides a reliable test of the calculation techniques of Chiral Perturbation Theory, our data disagree with the calculations.

PACS numbers: $13.60 . \mathrm{Hb}, 11.55 . \mathrm{Hx}, 25.30 . \mathrm{Rw}, 12.38 . \mathrm{Qk}, 24.70 .+\mathrm{s}$
\end{abstract}

\section{INTRODUCTION}

The Bjorken sum rule 1] relates an integral over the spin distributions of quarks inside the nucleon to its axial charge. This relation has been essential for understanding the nucleon spin structure and establishing, via its $Q^{2}$-dependence, that Quantum Chromodynamics (QCD) describes the strong force when spin is included. The Bjorken integral has been measured in polarized deep inelastic lepton scattering (DIS) at SLAC, CERN and DESY [4]- 7] and at moderate four-momentum transfer squared $Q^{2}$ at Jefferson Lab (JLab) [8], see e.g. Ref. [9] for a review. The variable $Q^{2}$ is inversely related to the space-time scale at which the nucleon is probed. In the perturbative QCD (pQCD) domain (high $Q^{2}$ ) the sum rule reads [10]:

$$
\begin{gathered}
\Gamma_{1}^{p-n}\left(Q^{2}\right) \equiv \int_{0}^{1} d x\left(g_{1}^{p}\left(x, Q^{2}\right)-g_{1}^{n}\left(x, Q^{2}\right)\right)= \\
\frac{g_{A}}{6}\left[1-\frac{\alpha_{s}}{\pi}-3.58 \frac{\alpha_{s}^{2}}{\pi^{2}}-20.21 \frac{\alpha_{s}^{3}}{\pi^{3}}+\ldots\right]+\sum_{i=2}^{\infty} \frac{\mu_{2 i}^{p-n}\left(Q^{2}\right)}{Q^{2 i-2}}
\end{gathered}
$$

where $g_{1}^{p}$ and $g_{1}^{n}$ are the spin-dependent proton and neutron structure functions, $g_{A}$ is the nucleon axial charge that controls the strength of neutron $\beta$-decay, $\alpha_{s}\left(Q^{2}\right)$ is the strong coupling strength and $x=Q^{2} / 2 M \nu$, with $\nu$ the energy transfer and $M$ the nucleon mass. The bracket term ( $\mu_{2}$, known as the leading twist term) is mildly dependent on $Q^{2}$ due to pQCD soft gluon radiation. The other term contains non-perturbative power corrections (higher twists). These are quark and gluon

\footnotetext{
*Present address: New Mexico State University, Las Cruces, NM 88003

${ }^{\dagger}$ Present address: Christopher Newport University, Newport News, VA 23606
}

correlations that need to be understood to describe the nucleon structure away from the large $Q^{2}$ limit. The $Q^{2}$-dependence of $\mu_{2 i}\left(Q^{2}\right)$ is calculable in principle from pQCD. In practice, this has been done for $\mu_{2}$ and $\mu_{4}$ only [11]. We stress that, as is almost always the case with $\mathrm{pQCD}$, although the $Q^{2}$-dependences are known, the absolute values of $\mu_{2}$ and $\mu_{4}$ are unknown and need to be measured or computed by non-perturbative means. Besides its contribution to establishing pQCD (at high $Q^{2}$ ), the Bjorken sum rule can be used to extract higher twists, to check lattice QCD calculations (at moderate $Q^{2}$ ), and to test effective theories of the strong force (at low $Q^{2}$ ). In addition, Bjorken sum data and phenomenological models at lower $Q^{2}$ can be described with a nearly constant "effective strong coupling" $\alpha_{s, g 1}[12,13]$. The lack of $Q^{2}$-dependence of $\alpha_{s, g 1}$ opens new avenues for non-perturbative QCD calculations using the AdS/CFT correspondence [14].

The elastic contribution to the Bjorken sum is usually not included because the generalized Bjorken sum rule is derived at large $Q^{2}$ where such contribution is negligible. Furthermore, the Bjorken sum rule naturally connects to the Gerasimov-Drell-Hearn sum rule [15] in which the elastic is inexistent. Consequently, when presenting the experimental measurement of the Bjorken sum, the elastic contribution will not be included. We refer to ref. [16] for a discussion on whether to include or not the elastic contribution to the GDH sum rule. However, for higher twist analysis, all reactions should be included for a meaningful higher twist extraction [16]-[18]. Therefore, in the part of the paper discussing higher twist extraction, the elastic contribution to the Bjorken sum will be added.

In this paper, new data from the JLab CLAS EG1b experiment [20]-22] taken on polarized proton and deuteron targets are used to extract the Bjorken integral over an extended $Q^{2}$ range: $0.05<Q^{2}<2.92 \mathrm{GeV}^{2}$ 
compared to the previous JLab range $0.15<Q^{2}<1.5$ $\mathrm{GeV}^{2}$ [8].

The extension down to $Q^{2}=0.05 \mathrm{GeV}^{2}$ allows us to compare to Chiral Perturbation Theory $(\chi P T)$ calculations in a domain where the chiral approximation should be valid. The moderate $Q^{2}$ range had been precisely measured [8]. The new data set, of equivalent precision, provides a useful check. In particular, it verifies the neutron results, which come mostly from ${ }^{3} \mathrm{He}$ in Ref. [8] and from the deuteron in this paper. At larger $Q^{2}\left(\gtrsim 1 \mathrm{GeV}^{2}\right.$, where Eq. (1holds), higher twists can now be studied with a statistical precision typically improved by a factor of 2 . Previous work [8] has shown the necessity of precise $Q^{2}$ mapping at moderate $Q^{2}\left(\gtrsim 1 \mathrm{GeV}^{2}\right)$ because of the surprisingly small size of the overall higher twist effect. One might be tempted to lower the $Q^{2}$ values at which the analysis is done (see Eq. 11) but this is not reliable due to the fast $1 / Q^{2 i-2}$ rise of twist $i$ contributions at low $Q^{2}$ and to the increasing uncertainty of the evolution of the twist-2 parts. The main contributor at low $Q^{2}$ to this uncertainty is the strong coupling constant $\alpha_{s}\left(Q^{2}\right)$.

The Bjorken integral is advantageous compared to the individual moments $\Gamma_{1}^{p}$ and $\Gamma_{1}^{n}$ because of simplifications arising from its non-singlet (p-n) nature: at moderate $Q^{2}$ lattice QCD calculations are easier and more reliable because disconnected diagrams, which cannot be easily computed on the lattice, cancel out. At higher $Q^{2}$, the (p-n) simplification provides a sum rule (the Bjorken sum rule) based on more solid grounds than the sum rules for individual nucleons (the Ellis Jaffe sum rules 19] that necessitate additional assumptions). At low $Q^{2}$, the $(\mathrm{p}-\mathrm{n})$ subtraction cancels the $\Delta_{1232}$ resonance contribution which makes the $\chi P T$ calculations significantly more reliable 35]. By a similar argument, the transverselongitudinal polarizability $\delta_{L T}[9]$, a higher moment of spin structure functions, also provides a reliable test of $\chi P T$ computations. (In that case, the $\Delta_{1232}$ contribution is suppressed at low $Q^{2}$ because the $\mathrm{N}-\Delta$ transition is mostly transverse, making the longitudinal-transverse (LT) interference term very small.) Nevertheless, calculations based on $\chi P T$ and data for $\delta_{L T}$ on the neutron [27] strongly disagree. This calls for more low $Q^{2}$ studies, especially the yet unmeasured $\delta_{L T}^{p}[36]$. The data discussed in this paper were taken with a longitudinally polarized target and hence cannot be used to extract $\delta_{L T}^{p}$. However, the generalized forward spin polarizability $\gamma_{0}$ can be obtained and, just like the Bjorken integral, its isovector part $\gamma_{0}^{p}-\gamma_{0}^{n}$ offers the same advantages as $\delta_{L T}$ for checking the calculation techniques of $\chi P T$. We will also report on these results.

\begin{tabular}{|c|c|c|c|c|c|}
\hline$Q^{2}$ & $\Gamma_{1, \text { meas }}^{p-n}$ & $\sigma_{\text {meas }}^{\text {syst }}$ & $\Gamma_{1, \text { tot }}^{p-n}$ & $\sigma^{\text {syst }}$ & $\sigma^{\text {stat }}$ \\
\hline \hline 0.054 & 0.0028 & 0.0105 & 0.0110 & 0.0119 & 0.0078 \\
\hline 0.078 & -0.0085 & 0.0112 & 0.0019 & 0.0134 & 0.0076 \\
\hline 0.101 & 0.0076 & 0.0105 & 0.0206 & 0.0134 & 0.0114 \\
\hline 0.132 & 0.0129 & 0.0124 & 0.0296 & 0.0158 & 0.0089 \\
\hline 0.188 & 0.0209 & 0.0181 & 0.0464 & 0.0223 & 0.0073 \\
\hline 0.268 & 0.0155 & 0.0152 & 0.0541 & 0.0218 & 0.0048 \\
\hline 0.382 & 0.0197 & 0.0139 & 0.0750 & 0.0229 & 0.0038 \\
\hline 0.496 & 0.0184 & 0.0110 & 0.0907 & 0.0225 & 0.0045 \\
\hline 0.592 & 0.0318 & 0.0143 & 0.1027 & 0.0228 & 0.0052 \\
\hline 0.707 & 0.0513 & 0.0174 & 0.0945 & 0.0201 & 0.0151 \\
\hline 0.844 & 0.0507 & 0.0157 & 0.1021 & 0.0193 & 0.0174 \\
\hline 1.01 & 0.0656 & 0.0152 & 0.1236 & 0.0200 & 0.0156 \\
\hline 1.20 & 0.0628 & 0.0161 & 0.1307 & 0.0192 & 0.0145 \\
\hline 1.44 & 0.0718 & 0.0141 & 0.1522 & 0.0186 & 0.0089 \\
\hline 1.71 & 0.0695 & 0.0129 & 0.1605 & 0.0182 & 0.0069 \\
\hline 2.05 & 0.0616 & 0.0118 & 0.1678 & 0.0177 & 0.0056 \\
\hline 2.44 & 0.0458 & 0.0098 & 0.1666 & 0.0167 & 0.0045 \\
\hline 2.92 & 0.0483 & 0.0079 & 0.1789 & 0.0106 & 0.0035 \\
\hline
\end{tabular}

TABLE I: The measured $\left(\Gamma_{1, \text { meas }}^{p-n}\right)$ and total $\left(\Gamma_{1, \text { tot }}^{p-n}\right)$ Bjorken integrals for different $Q^{2}$ points (in $\mathrm{GeV}^{2}$ ). The experimental systematic uncertainty $\sigma_{\text {meas }}^{\text {syst }}$ is given in the $3^{\text {rd }}$ column. Total systematics uncertainty, including the low and large- $x$ extrapolations, $\left(\sigma^{\text {syst }}\right)$ and statistical uncertainty $\left(\sigma^{\text {stat }}\right)$ on $\Gamma_{1, \text { tot }}^{p-n}$ are given in the $5^{\text {th }}$ and $6^{\text {th }}$ columns.

\section{BJORKEN SUM EXTRACTION AND COMPARISON WITH CHIRAL PERTURBATION CALCULATIONS}

The measurements of structure functions $g_{1}^{p}$ and $g_{1}^{d}$ are described in Refs. [20]-222]. The data cover an invariant mass range up to $W=3 \mathrm{GeV}$ for $0.054 \leq Q^{2} \leq$ $2.92 \mathrm{GeV}^{2}$. Since experimental moments are integrated over a finite $W$ range, the data have to be supplemented by models for large $W$. We used the model described in Ref. 20] down to $x=0.001$. This part is known from DIS experiments. The rest is determined using a Regge parametrization 8] which was compared to that of Bass and Brisudova [28] and found consistent with it. A parameterization was also used to estimate the contributions between pion threshold $(1.08 \mathrm{GeV})$ and 1.15 $\mathrm{GeV}$ 20].

The Bjorken integral is obtained from $\Gamma_{1}^{p}$ and $\Gamma_{1}^{d}$ assuming:

$$
\Gamma_{1}^{p-n}=2 \Gamma_{1}^{p}-\Gamma_{1}^{d} /\left(1-1.5 \omega_{d}\right),
$$

with the deuteron D-state probability $\omega_{d}=0.05 \pm$ 0.01 [29]. The data are given in Table I (a more detailed table is given in [30]) and shown in Fig. 1. The elastic contribution $(x=1)$ is excluded. Data from SLAC E143 [3], HERMES 7], JLab CLAS EG1a (proton and deuteron), and JLab Hall A E94010 (neutron from ${ }^{3} \mathrm{He}$ ) combined with CLAS EG1a (proton) [8] are also shown for comparison.

There is excellent agreement between the Bjorken inte- 


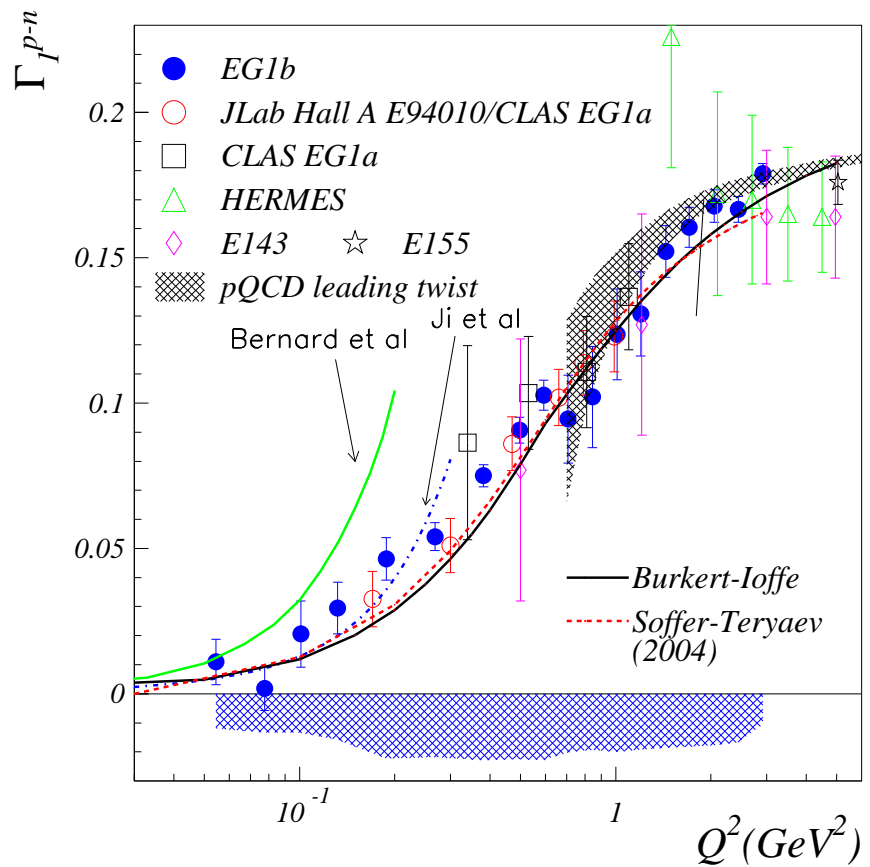

FIG. 1: (Color online) The Bjorken integral $\Gamma_{1}^{p-n}\left(Q^{2}\right)$. The solid blue circles give the results from this work with the horizontal band giving the systematic uncertainties. Other symbols show the data from experiments E143 [3] (open diamonds), E155 [5] (open star), HERMES [7] (open triangles) and JLab [8] (open circles and open squares). For those, the error bars represent the quadratic sum of the statistic and systematic uncertainties. The gray band represents the leadingtwist NNLO pQCD calculation. The curves correspond to $\chi P T$ calculations ( 33,34$])$ and phenomenological models ( 31,32$]$ ).

gral with the neutron extracted from the deuteron (filled circles and open squares) and from ${ }^{3} \mathrm{He}$ (open circles). The neutron spin structure functions extracted from the deuteron and from ${ }^{3} \mathrm{He}$ agree at moderate and large $Q^{2}$. However, for $Q^{2}$ below a few tenths of a $\mathrm{GeV}^{2}$, nuclear effects beyond those accounted for in the convolution method employed to extract the neutron [23] may become large 24]. Therefore, at low $Q^{2}$ one needs both the deuteron and ${ }^{3} \mathrm{He}$ data to ensure a reliable neutron extraction. Nuclear effects in the deuteron are weaker, but there is an unsuppressed contribution from the proton. On the other hand, ${ }^{3} \mathrm{He}$ is more tightly bound, but the polarized proton contribution is largely suppressed. Consequently, the uncertainty due to nuclear effects is mostly of different origin in the deuteron and ${ }^{3} \mathrm{He}$, which makes the two nuclei complementary. The agreement between the deuteron and ${ }^{3} \mathrm{He}$ results is also encouraging for the interpretation of the low $Q^{2}{ }^{3} \mathrm{He}$ and the deuteron data $\left(Q^{2}>0.015 \mathrm{GeV}^{2}\right)$ that will be available shortly respectively from Jefferson Lab's Hall A 25] and B [26]. The data also agree well with the SLAC and HERMES experiments and with the two phenomenological models shown in Fig. 11 The model of Burkert and Ioffe 31] (contin- uous black curve) is a meson-dominance-based extrapolation of DIS data supplemented by a parametrization of the resonance contribution. The other model (SofferTeryaev [32], dashed red curve) uses the smoothness of $g_{1}+g_{2}$ with $Q^{2}$ to extrapolate DIS data at lower $Q^{2}$.

At moderate $Q^{2}$, we observe a strong variation of the Bjorken integral, in contrast to the high $Q^{2}$ region. Together with our data at the lowest $Q^{2}$ points, the kinematic constraint $\Gamma_{1} \rightarrow 0$ when $Q^{2} \rightarrow 0$ suggests a small $Q^{2}$-dependence of $\Gamma_{1}^{p-n}$ at low $Q^{2}$ as well. This would agree with the fact that the $\Gamma_{1}$ slope at $Q^{2} \simeq 0$ is given by the generalized GDH sum rule which predict a small $Q^{2}$-dependence.

At low $Q^{2}$ the data are consistent up to $Q^{2} \simeq 0.2 \mathrm{GeV}^{2}$ with the $\chi P T$ calculations of Bernard et al. 33] and up to $Q^{2} \simeq 0.35 \mathrm{GeV}^{2}$ for those of Ji et al. done in the heavy baryon approximation 34]. The range of validity of the $\chi P T$ calculations seems larger than of individual nucleons [9], 22] possibly because the $\Delta_{1232}$ resonance is suppressed in the Bjorken integral [35]. This result, however, is not trivial: Good agreement was expected between $\delta_{L T}$ and $\chi P T$ results since the $\Delta_{1232}$ is strongly suppressed at low $Q^{2}$ for $\delta_{L T}$. However, its measurement for the neutron [27] disagrees strongly with $\chi P T$ calculations.

To quantitatively compare with $\chi P T$ calculations, we fit our results up to a maximum $Q^{2}$ ranging from 0.30 to $0.50 \mathrm{GeV}^{2}$ (fits on lower $Q^{2}$ ranges are imprecise, and higher $Q^{2}$ data may lie out of the region of validity for $\chi P T)$. We included the data from Ref. [8] in the fit. Our fit form is:

$$
\Gamma_{1}^{p-n}=\frac{\kappa_{n}^{2}-\kappa_{p}^{2}}{8 M} Q^{2}+a Q^{4}+b Q^{6}
$$

in which $\kappa$ is the anomalous moment of the nucleon and $a$ and $b$ are fit parameters. The first term in Eq. 2 stems from the Gerasimov-Drell-Hearn sum rule 9]. We find $a=0.80 \pm 0.07$ (stat) \pm 0.23 (syst) and $b=-1.13 \pm$ 0.16 (stat) \pm 0.39 (syst) with $\chi^{2} /$ dof $=1.50$. The $Q^{4}$ term agrees well with the results from Ji et al. $(a=0.74)$ but not with those of Bernard et al. $(a=2.4)$. The fit underscores the importance of the $Q^{6}$ term (not calculated yet in $\chi P T)$. This was also noticed for $\Gamma_{1}^{p}$ and $\Gamma_{1}^{d}[22]$.

At high $Q^{2}$, the leading twist pQCD calculation is given by the bracket term of Eq. 1 and is represented by the gray band in Fig. 1. It agrees reasonably well with the data. This implies that the total higher twist contribution is relatively small even down to $Q^{2} \approx 1 \mathrm{GeV}^{2}$ where one would expect higher twist contributions to be significant. Higher twists, which measure parton correlations, are weighted by $1 / Q^{(t-2)}$ (with $t$ being the twist number) and are related to the confinement mechanisms and to scattering off coherent quarks. Because of these reasons, it was initially expected that higher twists would play an important role at $Q^{2} \lesssim 1 \mathrm{GeV}^{2}$. Higher twists can be positive or negative but there is no fundamental reason to expect a well-tuned cancellation of different terms in 
the higher twist series that would make the overall higher twist contribution small. However, this seems to be the case experimentally, at least around $Q^{2} \approx 1 \mathrm{GeV}^{2}$. One of the aims of the higher twist analysis reported here is to establish whether higher twists are intrinsically small, or whether the terms in the higher twist series conspire to cancel.

\section{HIGHER TWIST ANALYSIS}

The first higher twist correction term in Eq. 11 is [1]]:

$$
\mu_{4}^{p-n}=\frac{M^{2}}{9}\left(a_{2}^{p-n}+4 d_{2}^{p-n}+4 f_{2}^{p-n}\right),
$$

where $a_{2}$ and $d_{2}$ are known. They are given by moments of the leading twist part of $g_{1}$ and the twists 2 and 3 parts of $g_{2}: a_{2}=\int_{0}^{1} d x\left(x^{2} g_{1}\right)$ and $d_{2}=\int_{0}^{1} d x x^{2}\left(2 g_{1}+3 g_{2}\right)$. The twist- 4 term that we wish to extract is $f_{2}^{p-n}$.

To perform a higher twist analysis, the elastic contribution $(x=1)$ to $\Gamma_{1}^{p-n}$ is added. The moment $\Gamma_{1}^{p-n}$ which includes the elastic contribution estimated from form factor parameterizations 37] is shown in Fig. 2. In Eq. 1. $\alpha_{s}$ is computed up to next to leading order. A fit of polar-

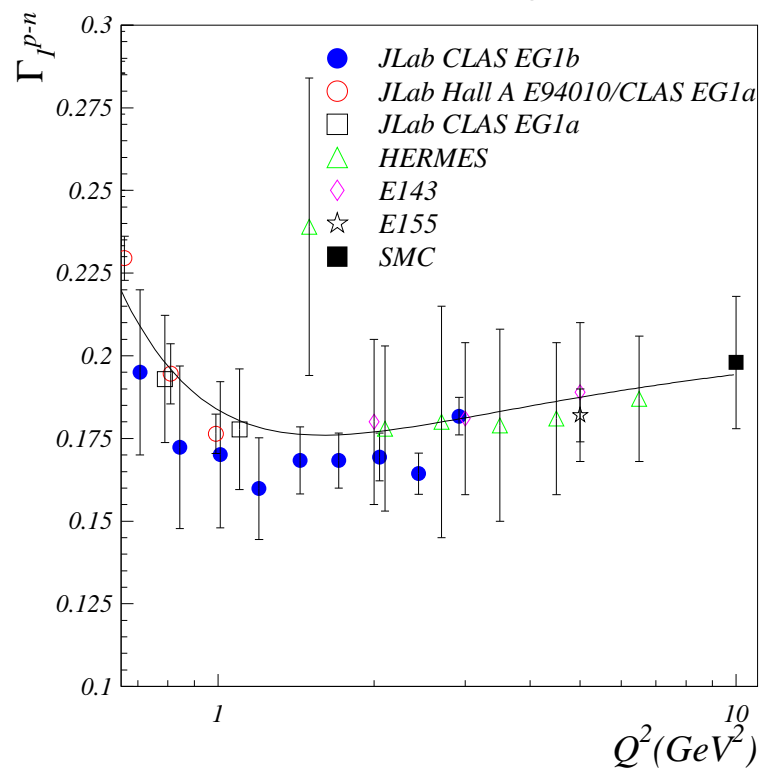

FIG. 2: (Color online) World data on the Bjorken integral, including the elastic contribution. The error bars represent the quadratic sum of the statistic and point-to-point-uncorrelated systematic uncertainties for the JLab data, and the quadratic sum of the statistic and full systematic uncertainties for the rest of the data. The continuous line is our three parameter fit in the $Q^{2}$-range from 0.66 to $10 \mathrm{GeV}^{2}$.

ized quark distributions [38] yields $a_{2}^{p-n}=0.031 \pm 0.010$ at $Q^{2}=1 \mathrm{GeV}^{2}$, whereas $d_{2}^{p-n}=-0.007 \pm 0.010$ is obtained from Ref. [5] and Ref. [39] evolved to $1 \mathrm{GeV}^{2}$. The EG1b data on $\Gamma_{1}^{p-n}$, together with the world's data, can then be fit to extract $f_{2}^{p-n}$ using Eqs. 11 and 3. To account for twists greater than rank 4, we include a coefficient $\mu_{6}^{p-n} / Q^{4}$. For consistency, former data on $\Gamma_{1}^{p-n}$ were reanalyzed using the same model as used in this paper to extrapolate to low $x$. For both JLab data sets (Ref. 8] and the present data), the point-to-point correlated uncertainties have been separated from the uncorrelated ones. The latter are added in quadrature to the statistical uncertainties. The correlated systematics are propagated independently, as is the uncertainty arising from $\alpha_{s}$. The result of the fit done in the $Q^{2}$-range from 0.66 to 10.0 $\mathrm{GeV}^{2}$ is $f_{2}^{p-n}\left(Q^{2}=1 \mathrm{GeV}^{2}\right)=-0.101 \pm 0.027 \pm_{0.071}^{0.063}$ with $\mu_{6} / M^{4}=0.084 \pm 0.011 \pm_{0.026}^{0.022}$. The first uncertainty is the quadratic sum of the statistical and the point-topoint uncorrelated uncertainties. The second one is the point to point correlated uncertainty. Comparing the values of $f_{2}^{p-n}, a_{2}^{p-n}$ and $d_{2}^{p-n}$ at $Q^{2}=1 \mathrm{GeV}^{2}$, we see that $\mu_{4}^{p-n} \approx 0.4 f_{2}^{p-n} \mathrm{GeV}^{2}$. The result for $f_{2}^{p-n}$ is plotted in Fig. 3 (square) along with the result from Ref. [8] (triangle) and theoretical predictions (In addition to $f_{2}$ and $\mu_{6}$, the third fit parameter mentioned in Figs. 2 and 3 is $g_{a}$, which was free to vary within its experimental uncertainty). As discussed in the introduction, only the $Q^{2}$-dependence of $f_{2}$ is known from pQCD. The absolute value can be computed solely from non-perturbative means and is difficult to obtain with Lattice QCD. For these reasons, only phenomenological models are available for comparison with our results.

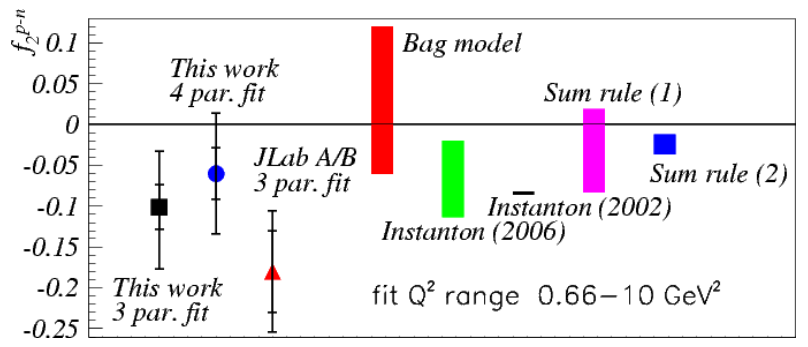

FIG. 3: $f_{2}^{p-n}\left(Q^{2}=1 \mathrm{GeV}^{2}\right)$ for the fits performed over the $0.66<Q^{2}<10 \mathrm{GeV}^{2}$ range for this study and Ref. [8] (JLab A/B). Calculations [40]- 44] are shown by the bands. Sum rule (1) refers to Ref [41] and (2) to Ref [40].

At $Q^{2}=1 \mathrm{GeV}^{2}$, the leading twist term $\mu_{2}^{p-n}$ and higher twist terms $\mu_{4}^{p-n}$ and $\mu_{6}^{p-n}$ are of similar sizes but with alternating signs and with $\mu_{4}^{p-n}$ and $\mu_{6}^{p-n}$ mostly canceling each other.

To study the systematics associated with this higher twist analysis and to check the legitimacy of our procedure at low $Q^{2}$, we conducted several tests:

1. We repeated the fit for several $Q^{2}$ ranges;

2. We reiterated this work adding a $\mu_{8}^{p-n} / Q^{6}$ term to study the convergence of the twist series (the resulting $f_{2}^{p-n}$ is shown in Fig. 3 by the solid circle);

3 . We investigated the dependence on the low $x$ extrapolation using different Regge-based parameterizations; 
4. We extensively studied the stability of the fit for different choices of number of parameters and of $Q^{2}$ ranges by using different models that reproduce the data reasonably well. We used ranges from $0.47<$ $Q^{2}<10$ to $3<Q^{2}<10 \mathrm{GeV}^{2}$ and we fit with functional forms with highest term from $\mu_{6} / Q^{4}$ to $\mu_{12} / Q^{10}$.

All observations supports the validity of our extractions. See Ref. [30] for details.

\section{COLOR POLARIZABILITIES}

Combination of higher twist coefficients can be interpreted in terms of color polarizabilities, which describe the response of the color magnetic and electric fields to the spin of the nucleon. The color electric and magnetic polarizabilities [40], [45] are $\chi_{E}=\frac{2}{3}\left(2 d_{2}+f_{2}\right)$ and $\chi_{B}=\frac{1}{3}\left(4 d_{2}-f_{2}\right)$. Using the value of $f_{2}^{p-n}$ extracted from the fit with $Q_{m i n}^{2}=0.66$, we obtain $\chi_{E}^{p-n}=$ $-0.077 \pm 0.050$ and $\chi_{B}^{p-n}=0.024 \pm 0.028$. The pointto-point correlated and uncorrelated uncertainties on $f_{2}$ were added in quadrature. Our higher twist analysis yields $\left|f_{2}^{p-n}\right| \gg\left|d_{2}^{p-n}\right|$ (a feature predicted by models [43] and [44]). Consequently $\chi_{E}^{p-n} \simeq \frac{2}{3} f_{2}^{p-n}$ and $\chi_{B}^{p-n} \simeq-\frac{1}{3} f_{2}^{p-n}$.

\section{ELECTROMAGNETIC POLARIZABILITY}

We now turn to the generalized forward spin polarizability $\gamma_{0}$. Spin polarizabilities characterize the coherent response of the nucleon to photons. They are defined using low-energy theorems in the form of a series expansion in the photon energy. The first term of the series comes from the spatial distribution of charge and current (form factors) while the second term results from the deformation of these distributions induced by the photon (polarizabilities). Hence, polarizabilities are as important as form factors in understanding coherent nucleon structure. Generalized spin polarizabilities describe the response to virtual photons. The low energy theorem defining the generalized forward spin polarizability is:

$$
\begin{array}{r}
\Re e\left[g_{T T}\left(\nu, Q^{2}\right)-g_{T T}^{p o ̂ l e}\left(\nu, Q^{2}\right)\right]= \\
\left(\frac{2 \alpha}{M^{2}}\right) I_{T T}\left(Q^{2}\right) \nu+\gamma_{o}\left(Q^{2}\right) \nu^{3}+O\left(\nu^{5}\right),
\end{array}
$$

where $g_{T T}$ is the spin-flip doubly-virtual Compton scattering amplitude, and $I_{T T}$ is the coefficient of the $O(\nu)$ term of the Compton amplitude which can be used to generalize the Gerasimov-Drell-Hearn $(\mathrm{GDH})$ sum rule to non-zero $Q^{2}[9,15]$. We have $I_{T T}\left(Q^{2}=0\right)=\kappa / 4$. In practice $\gamma_{0}$ can be obtained from a sum rule which has a derivation akin to that of the GDH sum rule:

$$
\gamma_{0}=\frac{16 \alpha M^{2}}{Q^{6}} \int_{0}^{x_{0}} x^{2}\left(g_{1}-\frac{4 M^{2}}{Q^{2}} x^{2} g_{2}\right) d x,
$$

where $g_{2}$ is the second spin structure function and $\alpha$ is the fine structure constant. Similar relations define the generalized longitudinal-transverse polarizability $\delta_{L T}$ :

$$
\begin{array}{r}
\Re e\left[g_{L T}\left(\nu, Q^{2}\right)-g_{L T}^{p \hat{o ̂ l e}}\left(\nu, Q^{2}\right)\right]= \\
\left(\frac{2 \alpha}{M^{2}}\right) Q I_{L T}\left(Q^{2}\right)+Q \delta_{L T}\left(Q^{2}\right) \nu^{2}+O\left(\nu^{4}\right), \\
\delta_{L T}=\frac{16 \alpha M^{2}}{Q^{6}} \int_{0}^{x_{0}} x^{2}\left(g_{1}+g_{2}\right) d x
\end{array}
$$

where $g_{L T}$ is the longitudinal-transverse interference amplitude, and $I_{L T}$ is the coefficient of the $O(\nu)$ term of the Compton amplitude. Details on the derivation of Eqs. 4,7 can be found in 9] and [46]. The isovector quantity $\gamma_{0}^{p}-\gamma_{0}^{n}$ eliminates the $\Delta_{1232}$ resonance contribution 35], and therefore offers the same advantage as $\delta_{L T}$ when comparing to calculations based on $\chi P T$. Higher moments are advantageous because they are essentially free of the uncertainty associated with the low $x$ extrapolation. An isospin separation of $\delta_{L T}$ or $\gamma_{0}$ may help us to understand why the $\chi P T$ calculations fail to describe them. For example, the $t$-channel exchange of axial-vector mesons (short range interactions), which are not included in the calculations, could be identified if one of the isospin components agrees with the $\chi P T$ calculations while the other disagrees.

We formed $\gamma_{0}^{p}-\gamma_{0}^{n}$ using the proton data from EG1b 22 and the neutron data from JLab experiment E94010 [27]. The ${ }^{3} \mathrm{He}$ data [27] are more precise than the deuteron data [22] that contain contributions from quasi-elastic and two-body break-up, which are not resolved by the CLAS spectrometer but are large at low $Q^{2}$. (This difficulty prevented $\gamma_{0}^{n}$ from being obtained from the EG1b data 22]). EG1b goes to lower $Q^{2}$ than E94010, but the coverage of E94010 is sufficient for our investigation. The resulting $\gamma_{0}^{p}-\gamma_{0}^{n}$ is shown in Fig. 目 (top plot) together with the predictions from Bernard et al. at $O\left(P^{4}\right)$ [33] and Kao et al. at $O\left(P^{4}\right)$ [47]. Experimental values are given in Table II. We also plot the result from the 2003 MAID model [48]. As is true for $\gamma_{0}^{p}$ [22] and $\gamma_{0}^{n}$ [27], $\chi P T$ calculations disagree with $\gamma_{0}^{p-n}$ as well. Clearly, the discrepancy seen for $\gamma_{0}^{p}$ and $\gamma_{0}^{n}$ cannot solely be due to the $\Delta_{1232}$ resonance. The MAID model, which provides a relatively good description of $\gamma_{0}^{p}$ and $\gamma_{0}^{n}$, disagrees mildly for their difference at the lowest $Q^{2}$ point. Complementary to this study, we formed the isoscalar part $\gamma_{0}^{p}+\gamma_{0}^{n}$ and compared it to the data (Fig. 4 bottom plot). The gray band on the Bernard et al. result is due to the uncertainty from the $\Delta_{1232}$ resonance. The MAID model provides a good description, whereas the $\chi P T$-based calculations still disagree. A disagreement in 


\begin{tabular}{|c|c|c|c|c|}
\hline$Q^{2}\left(\mathrm{GeV}^{2}\right)$ & $\gamma_{0}^{p-n}$ & $\gamma_{0}^{p+n}$ & Stat. & Syst. \\
\hline \hline 0.1 & 1.53 & -2.51 & 0.120 & 0.490 \\
\hline 0.26 & 0.470 & -0.869 & 0.021 & 0.177 \\
\hline 0.42 & 0.159 & -0.241 & 0.006 & 0.058 \\
\hline 0.58 & 0.0835 & -0.0845 & 0.0040 & 0.0233 \\
\hline 0.74 & 0.0441 & -0.0299 & 0.0037 & 0.0090 \\
\hline 0.9 & 0.0217 & -0.0103 & 0.0016 & 0.0040 \\
\hline
\end{tabular}

TABLE II: Isovector and isoscalar parts of the generalized forward spin polarizability $\gamma_{0}$.

the $\chi P T$ calculation of one of the isospin components of $\gamma_{0}$ along with agreement for the other component might have allowed us to identify a missing piece, such as for example a short range interaction due to heavy mesons, in the $\chi P T$ calculations. However, the discrepancy between data and $\chi P T$ calculations for both isospin components does not allow us to draw such conclusion. This suggests that the non-resonant background is responsible.

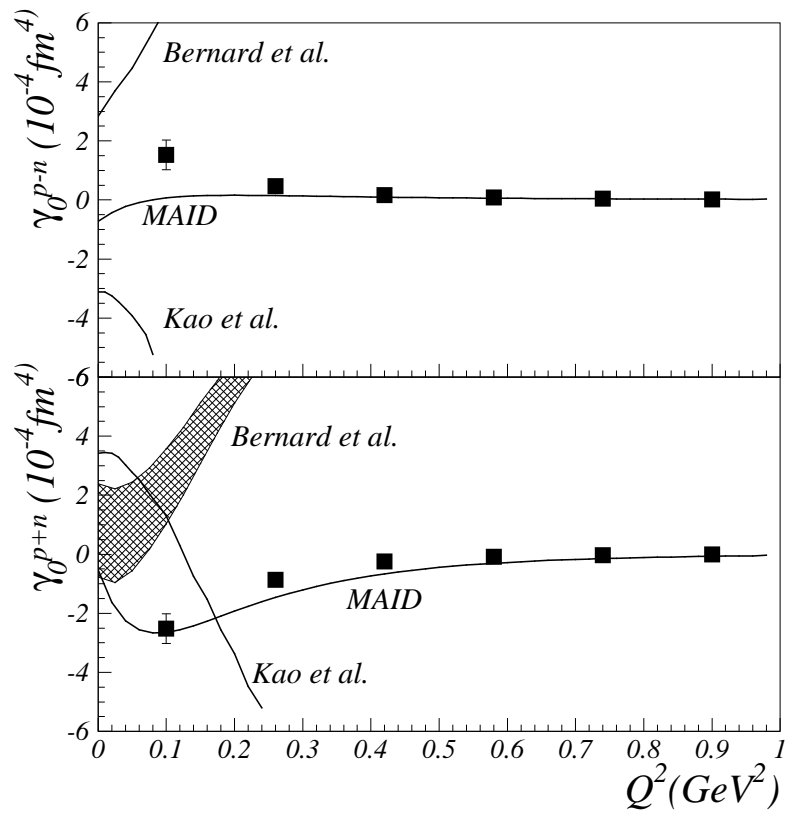

FIG. 4: The isovector $\gamma_{0}^{p}-\gamma_{0}^{n}$ (top) and isoscalar $\gamma_{0}^{p}+\gamma_{0}^{n}$ (bottom) generalized forward spin polarizabilities together with $\chi P T$-based calculations and the results from the MAID model. The proton and neutron data are respectively from CLAS 22] and Hall A [27]

\section{SUMMARY AND CONCLUSION}

The Bjorken integral was extracted from polarized proton and deuteron data for $0.054<Q^{2}<2.92 \mathrm{GeV}^{2}$. The results for intermediate $Q^{2}$ (the parton to hadron transition domain) are consistent with previous JLab data in which the neutron information was extracted from polarized ${ }^{3} \mathrm{He}$. This region exhibits a strong $Q^{2}$-behavior, both from $\mathrm{pQCD}$ evolution and from some higher-twist effects. On the other hand, in the high- $Q^{2}$ domain the
Bjorken integral is rather flat. The data together with kinematic constraints at $Q^{2} \rightarrow 0$ also suggest a small $Q^{2}$-dependence, in qualitative agreement with the generalized GDH sum predictions.

At the lowest $Q^{2}$ accessed by our data, $\chi P T$ calculations agree better with the Bjorken integral (an isovector quantity in which the $\Delta_{1232}$ resonance does not contribute) than with moments on individual nucleons. This is not trivial since the $\chi P T$ calculations fail to describe the generalized spin polarizability $\delta_{L T}$ in which the $\Delta_{1232}$ is also suppressed.

Data on the generalized forward spin polarizability $\gamma_{0}^{p-n}$ are not reproduced by the $\chi P T$-based calculations even though the $\Delta_{1232}$ does not contribute.

It is clear from previously published data on $\delta_{L T}$ and our analysis of $\gamma_{0}$ that the $\Delta_{1232}$ resonance contribution is not responsible for the discrepancy between data and calculations. The discrepancy between the $\chi P T$ calculations and the data occurs in all isospin channels, which makes it less likely that it is due to the contribution from heavier mesons in the chiral expansion.

The low $Q^{2} \chi P T$ regime has been recently mapped by two additional dedicated experiments in CLAS using polarized proton [49] and deuteron targets [26] and one in Hall A using polarized ${ }^{3} \mathrm{He}$ [25]. These experiments will provide further precision tests of $\chi P T$ calculation techniques.

The moderate $Q^{2}$ data $\left(1\right.$ to $3 \mathrm{GeV}^{2}$ ) allow us to extract higher twist contributions and color polarizabilities. The twist- 4 coefficient was found to be large: $f_{2}^{p-n} \simeq-0.1$ at $Q^{2}=1 \mathrm{GeV}^{2}$ (compare to $\Gamma_{1}^{p-n}=0.125$, $a_{2}^{p-n}=0.031$ and $\left.d_{2}^{p-n}=-0.007\right)$. The uncertainty on $f_{2}^{p-n}$ remains relatively large $(\approx 70 \%)$; however, we have completed several systematic studies both with the existing data as well as simulated data (with no statistic fluctuations) that indicate our result is stable. The sign and magnitude of $f_{2}^{p-n}$ agree with a recent analysis performed on $g_{1}$ directly [50]. The observation that higher twist effects on $\Gamma_{1}^{p-n}$ are small overall does not imply that the net higher twist effect on the structure function $g_{1}^{p-n}$ is small at any $x$. It is important to study the $x$-dependence of the higher twists, as is done in Ref. [50]. That $\left|f_{2}\right|$ is significantly larger than $d_{2}$, and that $f_{2}<0$, agrees well with the prediction of the twoscale model [44]. Overall the net effect of higher twists is small, because of a cancellation between the twist 4 and twist 6 terms that are of similar sizes but opposite signs. This trend has also been seen for higher twist analyses done on the unpolarized structure function $F_{2}$ [51]. This can be interpreted within a vector dominance framework: the oscillating signs arise from the development in series of the vector meson propagator $\propto 1 /\left(Q^{2}-M_{m}^{2}\right)$ where $M_{m}$ is the meson mass.

This work is supported by the U.S. Department of Energy (DOE) and the U.S. National Science Foundation. The Jefferson Science Associates operate the Thomas Jef- 
ferson National Accelerator Facility for the DOE under contract DE-AC05-84ER40150.

[1] J. D. Bjorken, Phys. Rev. 148, 1467 (1966); D 1, 465 (1970); Phys. Rev. D 1, 1376 (1970).

[2] E142 Collaboration, P. L. Anthony et al., Phys. Rev. Lett. 71, 959 (1993)

[3] K. Abe et al., Phys. Rev. Lett. 74, 346 (1995); 75, 25 (1995); 76, 587 (1996); Phys. Lett. B 364, 61 (1995); Phys. Rev. D 58, 112003 (1998).

[4] E154 collaboration: K. Abe et al., Phys. Rev. Lett. 79, 26 (1997).

[5] P. L. Anthony, et al., Phys. Lett. B 458, 529 (1999); B 463, 339 (1999); B 493, 19 (2000); B 553, 18 (2003).

[6] SMC collaboration: D. Adeva et al., Phys. Rev. D 58, 112001 (1998).

[7] HERMES collaboration: K. Ackerstaff, et al., Phys. Lett. B 404, 383 (1997); B 444, 531 (1998); A. Airapetian, et al., Phys. Lett. B 442, 484 (1998); Phys. Rev. Lett. 90, 092002 (2003); Eur. Phys. J. C 26, 527 (2003); Phys. Rev. D 75, 012007 (2007).

[8] A. Deur et al., Phys. Rev. Lett. 93, 212001-1 (2004).

[9] J.-P. Chen, A. Deur, Z.-E. Meziani; Mod. Phys. Lett. A 20, 2745 (2005).

[10] A. L. Kataev, Phys. Rev. D 50, R5469 (1994).

[11] E. Shuryak and A. Vainshtein, Nucl. Phys. B 201, 141 (1982); X. Ji and P. Unrau, Phys. Lett. B 333, 228 (1994); H. Kawamura et al. Mod. Phys. Lett. A 12, 135 (1997).

[12] A. Deur, V. Burkert, J.P. Chen and W. Korsch, Phys. Lett. B 650, 244 (2007).

[13] A. Deur, V. Burkert, J.P. Chen and W. Korsch. To be published in Phys. Lett. B. arXiv:0803.4119.

[14] See e.g. S. J. Brodsky and G. F. de Teramond, Phys. Rev. Lett 94, 201601 (2005); 96, 201601 (2006).

[15] S. D. Drell and A. C. Hearn, Phys. Rev. Lett. 16, 908 (1966). S. Gerasimov, Sov. J. Nucl. Phys. 2, 430 (1966).

[16] X. Ji, Phys. Lett. B 309187 (1993).

[17] X. Ji and W. Melnitchouk, Phys. Rev. D 56 R1 (1997).

[18] I. V. Musatov, O. V. Teryaev and A. Schafer. Phys Rev D 577041 (1998).

[19] J. R. Ellis and R. L. Jaffe Phys. Rev. D 9, 1444 (1974), Erratum-ibid. D 10, 1669 (1974)

[20] V. Dharmawardane et al., Phys. Lett. B 641, 11 (2006).

[21] P. E. Bosted et al., Phys. Rev. C 75, 035203 (2007).

[22] Y. Prok et al., arXiv:0802.2232

[23] See e.g. C. Ciofi degli Atti and S. Scopetta, Phys. Lett. B404, 223 (1997); M. Lacombe et al., Phys. Rev. C 21, 861 (1980)

[24] See e.g. A. Kievsky, E. Pace, G. Salmé, in Proceedings of the third symposium on the GDH sum rule and its extensions. World Scientific, J-P. Chen and S. Kuhn editors,
arXiv:nucl-th/0501060

[25] JLab experiment E97-110. J.-P. Chen, A. Deur, F. Garibaldi et al. www.jlab.org/exp_prog/proposals/97/PR97-110.pdf

[26] JLab experiment E06-017. A. Deur, G. Dodge, K. Slifer et al. www.jlab.org/exp_prog/proposals/06/PR06-017.pdf

[27] M. Amarian et al., Phys. Rev. Lett. 93152301 (2004).

[28] S. D. Bass, M. M. Brisudova, Eur. Phys. J. A 4, 251 (1999).

[29] M. Lacombe et al., Phys. Rev. C 21, 861 (1980); R. Machleidt, K. Holinde, C. Elster, Phys. Rept. 1491 (1987); M.J. Zuilhof, J.A. Tjon, Phys. Rev. C 22, 2369 (1980); K. Kotthoff, R. Machleidt, D. Schutte, Nucl. Phys. A 264, 484 (1976); B. Desplanques Phys. Lett. B 203, 200 (1988).

[30] A Deur, JLab report http://tnweb.jlab.org/tn/2008/08-001.pdf

[31] V. D. Burkert and B. L. Ioffe, Phys. Lett. B 296, 223 (1992); J. Exp. Theor. Phys. 78, 619 (1994).

[32] J. Soffer and O. V. Teryaev, Phys. Lett. B 545, 323 (2002); Phys. Rev. D 70116004 (2004).

[33] V. Bernard, T. R. Hemmert and Ulf-G. Meissner, Phys. Rev. D 67, 076008 (2003).

[34] X. Ji, C. W. Kao and J. Osborne, Phys. Lett. B 472, 1 (2000).

[35] V. D. Burkert, Phys. Rev. D 63, 097904 (2001).

[36] JLab experiment E08-027, K. Slifer, A. Camsonne, J-P. Chen et al. http://hallaweb.jlab.org/experiment/E07-001/docs/PAC33/dlt.pdf

[37] P. Mergell, U.-G. Meißner and D. Drechsel, Nucl. Phys. A596, 367 (1996).

[38] J. Bluemlein and H. Boettcher, Nucl. Phys. B 636, 225 (2002).

[39] Xiaochao Zheng et al. Phys. Rev. C70 065207 (2004).

[40] E. Stein et al., Phys. Lett. B 353, 107 (1995).

[41] I. Balitsky, V. Braun and A. Kolesnichenko, Phys. Lett. B 242, 245 (1990); Erratum-ibid B 318, 648 (1993).

[42] X. Ji and W. Melnitchouk, Phys. Rev. D 56, R1 (1997).

[43] N. Y. Lee, K. Goeke and C. Weiss, Phys. Rev. D 65, 054008 (2002).

[44] A. V. Sidorov and C. Weiss, Phys. Rev. D 73, 074016 (2006).

[45] X. Ji, hep-ph/9510362.

[46] D. Drechsel, B. Pasquini, M. Vanderhaeghen Phys. Rept. $\mathbf{3 7 8} 99$ (2003)

[47] C. W. Kao, T. Spitzenberg and M. Vanderhaeghen Phys. Rev D 67, 016001 (2003).

[48] D. Drechsel, S. Kamalov and L. Tiator. Nucl. Phys. A 645, 145 (1999).

[49] JLab experiment E03-006. M. Ripani, M. Battaglieri, A. Deur, R. DeVita, et al. www.jlab.org/exp_prog/proposals/03/PR03-006.pdf

[50] E. Leader, A. V. Sidorov, D. B. Stamenov. Phys. Rev D 75, 074027 (2007).

[51] M. Osipenko et al. Phys. Rev. D 67, 092001 (2003) 